\title{
La apropiación social de tecnologías móviles, una oportunidad para la formación ciudadana*
}

\author{
Beatriz Elena Marín Ochoa** \\ Luis Javier Cruz Lanchero ${ }^{* * *}$ \\ Recibido: 2020-09-02 • Enviado a pares: 2020-09-20 \\ Aprobado por pares: 2020-11-05 - Aceptado: 2021-01-16 \\ https://doi.org/10.22395/angr.v19n39a7
}

\begin{abstract}
Resumen
En la actualidad, las Tecnología de la Información y Comunicación (TIC) y los aplicativos móviles transforman la existencia de las personas; están presentes en todos los ámbitos y contextos cotidianos, de tal manera que ofrecen diversas perspectivas de abordaje para asumir retos como los que propone la educación en zonas rurales.

Con esta investigación se crea una propuesta de apropiación social de TIC por medio de estrategias de micro aprendizaje, mediados por dispositivos móviles para la formación ciudadana de estudiantes de grados decimo y once en una Institución rural del Urabá antioqueño.

La idea que surgió durante la ejecución del proyecto Ecosistema de Contenidos Digitales para Apps en Urabá, realizado entre 2019 y 2020, eligió a la Institución Educativa Rural Zapata (IERZ), que presta sus servicios en el corregimiento Zapata, Necoclí, para el trabajo de campo entre marzo y mayo de 2020, por el compromiso de la comunidad educativa y los avances en el proceso de Eduapps.

La metodología incluyó: una caracterización del nivel de apropiación frente a los dispositivos móviles y una categorización de cómo se evidencia la ciudadanía desde el ejercicio escolar cotidiano. Conocimientos que se transformaron en la propuesta de micro aprendizaje, modelo Eduapps, para fortalecer la formación ciudadana.

Como resultado, se logró demostrar que la propuesta promueve la formación y sintoniza la comunidad académica de la vereda, en función del uso de herramientas digitales móviles y apropiación de estrategias didácticas, para entender la importancia de asumir su propia ciudadanía con miras a construir un territorio en paz y con un real ejercicio de la democracia.

Palabras claves: aprendizaje, dispositivos móviles, educación ciudadana, enseñanza, micro aprendizaje, sociedad de la información, tecnologías de información, transferencia de conocimientos.
\end{abstract}

Este artículo es resultado del trabajo de grado Propuesta de Apropiación social de TIC por medio de micro-learning para la formación ciudadana - Aplicativo Edu-apps de la Maestría en Comunicación Digital de la Universidad Pontificia Bolivariana y como parte de la investigación Ecosistema de contenidos digitales para Apps en Urabá, Convocatoria 804 Regalías Antioquia - Min ciencias Contrato No. 80740 021-2019. Colombia.

. Doctora en Comunicación y Periodismo, Universidad Autónoma de Barcelona, España. Líder GI en Comunicación Urbana, Universidad Pontificia Bolivariana, Medellín, Colombia. Correo electrónico: beatrize.marin@upb.edu.co Orcid: https://orcid.org/0000-0001-6775-6636

... Politólogo de la Universidad Nacional de Colombia. Estudiante Maestría en Comunicación Digital Universidad Pontificia Bolivariana, Medellín, Colombia. Correo electrónico: luisjavier.cruz@upb.edu.co Orcid: https://orcid.org/0000-0002-9282-6015 


\title{
The Social Appropriation of Mobile Technologies: an Opportunity for the Construction of Citizenship
}

\begin{abstract}
Today, ICTs and mobile applications transform people's lives. They are present in all areas and everyday contexts, in such a way that they offer different perspectives and approaches to take on challenges such as those proposed by education in rural areas.

With this research, a proposal is created for the social appropriation of ICTs through micro-learning strategies mediated by mobile devices for the citizenship training of 10 th and 11 th-grade students in a rural institution in Urabá Antioqueño.

The idea that arose during the execution of the project "Ecosystem of Digital Content for Apps in Urabá", carried out between 2019 and 2020, was chosen by the Zapata Rural Educational Institution, IERZ, which provides its services to the Zapata district, Necoclí, for fieldwork between March and May 2020, for the commitment of the educational community and the advancements in the Eduapps process.

The methodology included: characterization of the level of appropriation in the face of mobile devices and categorization of how citizenship is evidenced from the daily school exercise. The knowledge was transformed into the micro-learning proposal, the Eduapps model, to strengthen citizen training.

In the end, it was possible to demonstrate that the proposal promotes training and tunes the academic community of the village based on the use of mobile digital tools and appropriation of didactic strategies, to understand the importance of assuming their own citizenship to build a peaceful territory and with a real exercise of democracy.
\end{abstract}

Keywords: learning; mobile devices; citizenship education; teaching; microlearning; information society; information technology; knowledge transfer.

\section{A apropriação Social das tecnologias móveis, uma oportunidade para a formação cidadã}

\begin{abstract}
Resumo
Atualmente, as Tecnologia da Informação e Comunicação (TIC) e os aplicativos móveis transformam a existência das pessoas; estão presentes em todos os âmbitos e contextos diários, de tal forma que oferecem várias perspectivas de abordagem para assumir desafios como os que propõem a educação nas zonas rurais.

Com esta investigação é criada uma proposta de apropriação social das TIC através de estratégias de micro aprendizagem, mediados por aparelhos móveis para a formação cidadã dos estudantes do ensino médio em uma Instituição rural de Urabá antioquenho.
\end{abstract}

A metodologia incluiu: caracterização do nível de apropriação frente aos aparelhos móveis e uma classificação do que é percebido os cidadãos a partir do exercício escolar diário. Também foi incluído conhecimentos que transformaram-se em proposta de micro aprendizagem, modelo Eduapps, para reforçar a formação cidadã.

Em consequência, foi possível mostrar que a proposta promove a formação e sintoniza a comunidade acadêmica da zona, com base ao uso de dispositivos digitais móveis e o uso de estratégias didáticas, para compreender a importância de aceitar sua condição de cidadão tendo em vista construir um território de paz com um verdadeiro exercício da democracia.

Palavras chave: aprendizagem, aparelhos móveis, educação cidadã, ensino, micro aprendizagem, sociedade da informação, tecnologias da informação, transferências de conhecimentos. 


\section{Introducción}

En la actualidad, las Tecnologías de la Información y Comunicación (TIC) y los aplicativos móviles, transforman la manera en que las personas existimos, gracias a su particular presencia en todos los ámbitos de la vida.

Por ello, y después de realizar un análisis de las dinámicas en la sociedad mediatizada, desde una perspectiva político-educativa, y a través de la ejemplificación de algunos trabajos de investigación aplicada en contextos escolares mediados por TIC, se estructuró una propuesta de intervención', desde la comunicación política² a partir de la pregunta: ¿̇es posible crear una propuesta de apropiación social de TIC mediado por el uso de dispositivos móviles que permita la formación ciudadana de estudiantes en una institución educativa rural?

El propósito era seguir el modelo de Edu-apps, macroproyecto en el que se inscribía esta propuesta, para generar apropiación social de TIC con estrategias de microlearning ${ }^{3}$ entre estudiantes de grados decimo y once del Urabá antioqueño, teniendo en cuenta que desde los inicios de los estudios conceptual y contextual de las TIC, las sociedades de la información y del conocimiento, facilitan la comprensión de las redes que configuran discursos, narraciones y experiencias (Benjamín, 2009), facilitan el entendimiento de la apropiación social de las TIC y la identificación de actores en la formación ciudadana o en la educación para la democracia (Gaviria, 2015).

Por lo anterior, se estableció como objetivo: crear una propuesta de apropiación social de TIC, por medio de microlearning, mediado por el uso de dispositivos móviles con el modelo Edu-apps, que permitiera la formación ciudadana en los estudiantes de los grados decimo y once en la IERZ, en el municipio de Necoclí (Antioquia). Para ello se requería: identificar niveles de apropiación social de TIC en aprendizaje móvil, determinar la formación ciudadana, a partir de la experiencia cotidiana, y plantear el diseño de una propuesta de apropiación social.

Por eso las reflexiones aquí presentes ofrecen perspectivas de abordaje para asumir retos sociales mediados por TIC, y brindar posibilidades a través del aprendizaje móvil para la formación ciudadana de jóvenes en proceso de finalización de la básica secundaria de una institución educativa en el municipio de Necoclí.

\footnotetext{
La denominación "Propuesta de intervención educativa" se refiere a una estrategia de planeación y actuación que permite a los docentes asumir el control de su propia práctica profesional mediante un proceso de indagación-solución.

2 Se plantea desde la comunicación política, porque la formación inicial del coautor y estudiante de Maestría es en ciencias políticas, y el programa en el que realiza el trabajo de grado es la Maestría de Comunicación Digital. Se observó un interés por aplicar el ejercicio de micro aprendizaje en un tema de encuentro entre ambas disciplinas.

3 Microlearning o micro aprendizaje es una forma de aprendizaje virtual a través de cursos cortos, mediante metodologías que combinan aprendizaje y estrategias de juego con la idea de ser un aprendizaje sencillo, rápido y efectivo.
} 
Afrontar los retos sociales de los últimos años y llegar a consensos para la cooperación internacional, las políticas públicas, la academia y otros sectores que inciden mediante redes de desarrollo cultural, científico, tecnológico en lo social, o en el potencial humano congregado en la "sociedad del conocimiento" o "sociedades del saber", requiere generar una concepción del desarrollo que va más allá de lo económico. Para la Unesco (2015), "una sociedad del conocimiento es una sociedad que se nutre de sus diversidades y capacidades (...) comprende dimensiones políticas, éticas y sociales" (p. 17).

En la actualidad, esto implica entender las dinámicas de interconexión política, económica, cultural y, sobre todo, tecnológica, que caracteriza al mundo en el siglo XXI, y que tiene que ver con los "dispositivos", más allá de aparatos tecnológicos. Un "dispositivo" o "los dispositivos". Según Foucault (1980), sobrepasan la dimensión del objeto físico, concentrando en sí la operatividad y, al mismo tiempo, la esencialidad de la realidad. Son representaciones concretas del "poder hacer", en una red compuesta por discursos, leyes, reglas, instituciones con una función estratégica que integra las relaciones de fuerza y lucha de contrarios, resultado del cruce entre la "relación del saber" y la "relación del poder". Por lo tanto, "un dispositivo puede ser entendido como una mediación donde se establece el diálogo constante entre las relaciones de poder y saber" (Claros, 2013, p. 6).

Esta relación que presenta el dispositivo pasa por la definición tecnológica y propicia que la materialización sea fácil de identificar en un simple y generalizado artefacto móvil, con el que, de forma masiva, los individuos y sujetos globales de hoy acceden a la web, se comunican, hacen negocios, trabajan o estudian. Además, los dispositivos están diseñados en múltiples niveles, a partir de la funcionalidad básica que permite que sean usados en movimiento (Nkeze, Pearce y Womer, 2007) o como combinación de telefonías móviles con funcionalidades agregadas de conectividad inalámbrica e internet (Nordin, Amin y Yunus, 2010), escenarios sociales o contextuales donde son considerados de manera exclusiva como artefactos predeterminados para una función específica, la realización de tareas o apoyos técnicos en la mayoría de los casos.

De acuerdo con el lingüista holandés, precursor del análisis del discurso como disciplina, Van Dijk (2005), la sociedad que se construye no es distinta a una colectividad que se configura y se entreteje desde una red que intenta abordar diversos aspectos, como la hiperconexión y el desarrollo, motivando el acceso y el uso del medio digital en todos los géneros y en todas las edades:

las redes se están convirtiendo en el sistema nervioso de nuestra sociedad y podemos esperar que esta infraestructura tenga más influencia en nuestra 
vida social y personal que la que tuvo la construcción de caminos para la transportación de personas y bienes en el pasado. (Van Dijk, 2005, p. 25)

Si se focaliza la atención en un espacio como el aula de clase, se puede encontrar que tanto alumnos como docentes dividen su atención entre lo que sucede en el salón y lo que interrumpe o se reclama desde sus celulares, siendo estos "más que un instrument (...) un escenario simbólico que involucra nuevas formas de sociabilidad y entretenimiento, para acercar la presencia de los otros, tener un status(sic) en el grupo de referencia" (Gewerc et al., 2017, p. 182).

Estas dinámicas afectan al tiempo ámbitos como los límites del espacio y el tiempo, pues si seguimos en el mismo ejemplo, la frontera entre lo escolar y lo no escolar ya no se define por el tiempo que toma ir hacia o desde la escuela, o el tiempo que se permanece en ella como lugar, un límite cada vez más difícil de precisar (Dussel y Quevedo, 2010).

De modo que, pretender abordar complejidades como la formación ciudadana, sin pensar en las TIC, restringe las posibilidades de desarrollo de habilidades sociales, la formación ética y moral, el crecimiento personal y la comprensión de los problemas y temas emergentes como: el medioambiente, los derechos humanos, la tolerancia, la discriminación, la diversidad cultural y social, la sexualidad y el género, la violencia, entre otros aspectos, los cuales entran a disputar los espacios, tiempos y definiciones de las subjetividades y cotidianidades, que en el ámbito educativo se refieren a la oferta curricular disponible, respecto a los contenidos y medios pedagógicos. Para ello es importante aclarar tres conceptos y establecer su relación: apropiación social de TIC, educación democrática y formación ciudadana, y aprendizaje móvil en la formación ciudadana.

\subsection{Apropiación social de TIC}

La apropiación se puede entender como el proceso de dominio de un artefacto técnico, práctica cotidiana reconocida y con efectos en el contexto social, a tal punto de provocar la regulación estricta por el Estado en algunos casos. Para Gómez Mont (2009), en comunicación el término busca "explicar el proceso de interiorización progresiva de competencias técnicas y cognitivas de individuos y grupos que manejan cotidianamente las TIC" (p. 8) y desde la visión de cultura tecnológica podemos decir que:

El desafío para el fortalecimiento de una cultura tecnológica y de un adecuado tránsito a una sociedad del conocimiento es que la gente ejerza sus capacidades para generar, apropiarse y aprovechar el conocimiento, tanto del tradicional como del científico, pero sobre todo que pueda generar el conocimiento que mejor le sirva para alcanzar sus fines. (Olivé, 2007, p. 73) 
Por lo anterior, la apropiación social del conocimiento de igual manera constituye un desafío en sí mismo, dado que implica comprender cómo se generan, integran y transforman las ideas desde las características de un determinado grupo social, en un momento específico y ante una escala de relaciones simbólicas. Es precisamente lo que en educación se conoce como un proceso multifacético, pues:

La apropiación posee varios niveles: a) el acceso a la información, al dato simple y escueto que circula por nuestras manos; b) la comprensión y correcta contextualización de cada dato que llega hasta nosotros; c) la reacción o construcción de una visión crítica ante el dato que se presenta y d) la posibilidad de compartirlo a través de redes sociales o redes digitales con el objeto de darle vida y renovarlo". (Gómez Mont, 2015, p. 2)

Llegados a esta complejidad de circuitos desde la concepción del conocimiento hasta sus prácticas sociales mediadas por TIC, es importante considerar la relación entre el saber-poder, y la ejecución de diferentes acciones independientes del tiempo y el lugar.

Es observable que el principal uso que dan los profesores a las TIC se resume en la proyección de videos, diapositivas e imágenes, y en la sistematización de notas o informes por medio de procesadores. De ahí que se puede concluir que la mayoría de los agentes educativos aún "se encuentran en un acercamiento a las TIC y realizan usos sencillos con ellas" (Cardona, Chaverra y Restrepo, 2016, p. 141), es decir, que su competencia tecnológica está en un nivel básico o explorador, según los niveles propuestos por el Ministerio de Educación Nacional (2013), y que el reto para que las TIC puedan mediar cada vez más y de mejor manera los contextos educativos y formativos, consiste en la apropiación social de sus actores, en nuestro caso de análisis: los docentes.

Las TIC ofrecen en un plano educativo de múltiples funciones, no solo de carácter pedagógico (como la creación de entornos más flexibles para el aprendizaje o el trabajo autónomo), sino en cuanto al desplazamiento del espacio y el tiempo, de manera sincrónica y asincrónica.

Esto con el fin de reducir las implicaciones negativas y favorecer, entre otros aspectos, las dinámicas colaborativas, colectivas, cocreativas y de equipo, por la facilidad con que favorecen la construcción de relaciones de y entre instituciones, programas, contenidos, docentes, estudiantes, padres de familia y comunidad. Lo anterior, en la medida en que la comunicación solo cambia según la modalidad de mediación utilizada, llámese redes sociales, foros o seminarios completos y en espacios virtuales que van desde plataformas web a aplicaciones móviles. 


\subsection{Educación democrática y formación ciudadana}

En este panorama, las reflexiones sobre la comunicación, las relaciones de poder presentes en el uso de las TIC, donde no solo tienen injerencia "con la producción de sentido, sino también con la estructuración de la sociedad" (Fuentes, 2010, p. 46), además de los nuevos contextos implícitos a los sujetos virtuales, nos arroja un sinfín de preguntas problematizadoras para múltiples investigaciones posibles, pero, sobre todo, reclama a la academia y a los profesionales, incidencia social para mediar las necesidades focalizadas y regionales de acuerdo a la problemática social colombiana.

Por otro lado, detenidos en la formación ciudadana, las posibilidades de intervención encuentran un nicho tecnológico fructífero según experiencias, como las referenciadas y citadas desde bases de datos de producciones académicas multidisciplinares y las indagaciones registradas para este texto.

La formación ciudadana se refleja en "un ser capaz de cooperar, dialogar y ejercer sus responsabilidades; un ciudadano que además de poseer unos derechos, también ejerza unos deberes, ya que democracia significa demos, y pueblo significa gente unida en torno a unos valores comunes" (Maldonado, 2004, p. 470). Este sujeto, en el ejercicio del encuentro cívico, representa una persona que se cuestiona sobre el manejo de lo público, pero no desde la escala del gobernante, sino desde la construcción colectiva y de cómo cada sujeto político impacta la realidad que habita.

La formación de ciudadanías o la pedagogía de la democracia, se vincula de forma íntima con el concepto de educación, cuya misión es: "entregar un conocimiento, contribuir al desarrollo de actitudes y, por, sobre todo, reforzar en los estudiantes aquellas habilidades sociales que les permiten relacionarse como ciudadanos de una misma humanidad" (Magendzo, 2005, p. 107). De suerte que la educación orienta a los sujetos, sus objetos y subjetividades hacia la productividad y la autonomía.

En el rumbo de posibilitar y propugnar por una formación ciudadana "actualizada" y contextualizada, la investigación y las competencias digitales en la escuela Boris (2015) afirma que:

las TIC han venido a la escuela para quedarse. No podemos concebir una escuela actual en la que los dispositivos electrónicos y la conectividad a Internet no formen parte del ecosistema escolar. Si la sociedad es con TIC, las escuelas deberían ser con TIC. (Boris, 2015, p. 77)

Como menciona Viñals (2016), las TIC están tan arraigadas en la sociedad de hoy que hasta se detectan en el "ocio". Así, dice Viñals (2016) en el estudio El ocio conectado, móvil, transmedia y multisoporte de los jóvenes en la era digital, que el desarrollo constante de las tecnologías digitales transforma en gran medida los ámbitos de actuación o 
actividad humana configurando las vivencias, pero también los momentos de ocio. Durante el año 2020, las personas invirtieron más tiempo de lo normal en las ofertas de ocio que le proponía el celular, pero el ocio como espacio de abandono propicia que se descubran momento de lucidez que facilite descubrir las bondades de potenciar las habilidades del "saber ser" y el "saber hacer".

Por su parte, Walter Benjamin (2009) de la Escuela de Fráncfort, plantea una teoría crítica de la comunicación y de su alcance social e histórico en su texto El Narrador que, similar a cómo ocurrió en el Oscurantismo Medieval, hace que la modernidad someta a la humanidad a una oscuridad no del conocimiento como en la Edad Media, sino de la experiencia. Lo que genera que cada vez más las personas deseen tener sensaciones experienciales; la realidad virtual ha propiciado su desarrollo y hoy las personas aprovechan esa tecnología porque hay que vivirlo, ya no es suficiente que le cuenten.

Dice Benjamín (1936) que las voces colectivas, autores y autoras, en la reproductibilidad técnica, es decir, en "quitarle su envoltura a cada objeto, triturar su aura, es la signatura de una percepción cuyo sentido para lo igual en el mundo ha crecido tanto que incluso, por medio de la reproducción, le gana terreno a lo irrepetible" (p. 5), al tratarse de un ejercicio de interacción desde lo informativo, el mensaje debe tener un lenguaje claro y sencillo que llegue al consumidor pertinente, para ello el emisor debe desprenderse de su dimensión subjetivo histórica, política y cultural. Esta "crisis de la experiencia" puede profundizarse en el consumismo exacerbado de información y en la alienación de los usuarios de internet y de TIC, pero, si como vemos no es posible mediar, sino contextualizar a través de diferentes dispositivos, la apropiación social resultante es la generación de experiencias, donde los sujetos digitales constituyen entramados desde su contexto real. Las narraciones en red que constituyen las sociedades del conocimiento, permiten generar experiencias a partir de la interacción con otros sujetos (Benjamín, 2009).

De manera que, si tenemos un contexto de generación de experiencias, las narraciones posibles se establecen en los contenidos mediados por las TIC y los dispositivos móviles. En esa línea, la educación democrática encuentra una muy amplia posibilidad de foro social y una cercanía con los contextos, comunidades y problemas sociales nunca antes posible gran escala.

La formación ciudadana entendida, como ya vimos, desde el encuentro cívico y la formación de nuevas ciudadanías, abre otros nichos que Carlos Gaviria Díaz (2015) incluía en lo que definía como "educar para la democracia". El maestro Gaviria fue uno de los demócratas más importantes en las últimas décadas en Colombia, no solamente desde la política, las altas cortes o el derecho, sino desde las transformaciones 
urgentes y necesarias de la sociedad. En la última conferencia que dio en el Gimnasio Moderno de Bogotá, el 11 de marzo de 2015, pocos días antes de morir, expuso las necesidades y desafíos de la democracia y de la educación, discutió las bases filosóficas de la cultura democrática, que sustentó en el liberalismo kantiano, al exponer y contextualizar ideas morales básicas como la tolerancia, la libertad, el pluralismo y la diversidad, el uso público de la razón en la constitución del Estado Social de Derecho y las garantías a las diferencias de convicción moral, opinión política y formas de vida estipulada en los derechos, y la Ley, en una Colombia de transformación cultural y política, por medio de la soberanía, la paz y la individualidad (Gaviria, 2015).

Estos precedentes filosóficos tienen arraigo en cualquier planteamiento actual sobre la educación en la constitución de ciudadanías, como lo plantean las profesoras Ruth Elena Quiroz Posada y Orlanda Jaramillo (2009), cualquier propuesta de formación ciudadana o educación para la democracia, debe basarse en unos objetivos o postulados mínimos para garantizar, por un lado, el componente educativo y, por otro lado, atender las necesidades urgentes de forjar ciudadanías que desarrollen y amplíen el espectro democrático. Estos mínimos serían:

- Conocer los derechos y ser responsable en el cumplimiento de las obligaciones, siendo cooperativo y plural.

- Fortalecer la identificación con los valores, principios y tradiciones que caracterizan al contexto global.

- Respetar la diversidad cultural de la humanidad, comprendiendo las diversas manifestaciones del pensamiento.

- Poner en ejercicio sus derechos y reconocerlos a los demás.

Indudablemente, estos retos permiten afirmar que una de las grandes tareas que debe cumplir la escuela es lograr que los estudiantes aprendan a desarrollar sus derechos como ciudadanos en el ámbito de lo cotidiano, porque es una institución de carácter público, colectivo, de reglamentación y de convivencia (Quiroz y Jaramillo, 2009, p. 111). El aporte de estos elementos a la formación ciudadana precisa unas coordenadas para enmarcar la lectura social y definir qué se busca aportar mediante las indagaciones previas a un proceso de investigación cualquiera.

En este caso, buscar la mediación de las TIC en la formación ciudadana, como experiencia que materialice una zona y poblaciones específicas, requiere exaltar lo cívico y lo ético como facultades ciudadanas para el buen vivir ("saber ser", "saber hacer"), en un contexto social, en el cual la tradición y la cultura moderen la creación de herramientas o tecnologías que se implementan para un impacto favorable 
a problemas sociales, comunitarios y regionales, donde el énfasis está puesto en las nuevas ciudadanías que plantea la necesidad de una formación democrática para la juventud.

\subsection{Aprendizaje móvil en la formación ciudadana}

Si la formación ciudadana es atravesada por el aprendizaje móvil, se deberá centrar en la forma tecnológica que la soporta. En este caso ya no sería el contexto donde ocurre el proceso de aprendizaje el principal componente, sino el artefacto facilitador del mismo. Algunos autores plantean que el aprendizaje móvil lo definen las tecnologías utilizadas para la adquisición de habilidades, es decir, que el proceso de aprendizaje se lleva a cabo a través de tecnologías inalámbricas, Wireless y dispositivos móviles como palms, tablets o smarthphones. Sin embargo, limitar el aprendizaje móvil al uso de tecnologías móviles, deja por fuera las bases del concepto en cuanto al contexto y a los contenidos educativos que participan en el acto educativo. Un problema recurrente, como ocurrió con la aparición del walkman en 1979 y con el uso de las cintas portables que permitían intercambiar los contenidos y tecnologías para fortalecer estrategias de aprendizaje de idiomas basados en audio. Al asignar otros contextos, hacerlos flexibles o virtuales, el aprendizaje se convierte en el contexto intervenido.

Este tipo de soporte para el aprendizaje proveía los contenidos necesarios para el desarrollo del mismo. El walkman, podría verse como un tipo de soporte que hace posible el aprendizaje por recepción y repetición (Díaz Barriga y Hernández, 2001). Esto, sin contar con los actuales mecanismos de interacción social sincrónica, ni mucho menos de interacción para la realización de actividades sobre el mismo soporte, como pasa hoy con aplicativos móviles en tiempo real, localización satelital y recursos de cámara o recepción de estímulos.

De lo anterior, se desprende que el celular, como contexto y dispositivo, es transversal a la cotidianidad humana, sobre todo en los jóvenes. Por ende, se puede tomar como aliado en los procesos de aprendizaje y formación, o darle una carga negativa y generar un hecho de desvinculación o distracción política, convirtiéndolo en un hecho que desarticula los sujetos con relación a su contexto real. Una mejor opción es tomar al dispositivo como mediador, es decir, como aliado, no solo por su uso cotidiano sino para potenciar el conocimiento.

En el análisis desde las sociedades del conocimiento en la globalización, la formación ciudadana y la contextualización de las TIC, se puede considerar el tránsito de la identificación de ese sujeto de la formación ciudadana mediada por las tecnologías móviles, a la identificación de la población objetivo en jóvenes escolarizados de los grados decimo y once. En la medida en que, con experiencias como las obte- 
nidas en Ciudadanía móvil: apropiación y participación de jóvenes en sectores vulnerables en Colombia, desarrollada en el 2010, cuyo objetivo fue analizar la participación ciudadana a través de la telefonía móvil en los jóvenes de sectores sociales vulnerables en el país, se puede avanzar con mayor confianza a partir de las subjetividades de los sujetos, en el abordaje de la apropiación social de la población objetivo, entendida como aquellos jóvenes vulnerables entre catorce y veintiséis años de edad, y que aparecían registrados en el Sisbén uno y dos de la ciudad de Barranquilla (Said, 2012).

De forma que, no basta con determinar que la formación ciudadana debe dirigirse a jóvenes vulnerables de grados decimo y once, y una metodología de selección de quienes en el tiempo adquirirán la investidura de ciudadanos, si no partimos de la importancia de que sean de municipios pertenecientes a las áreas con mayor rezago tecnológico y convulsión social, pues solo allí son apropiados los recursos y la generación de impacto esperada.

Sobre el aprendizaje móvil, vale la pena volver sobre el trabajo de Zambrano (2012), que en su libro Modelo de aprendizaje virtual, muestra cómo en algunos países europeos los modelos pedagógicos "se basan en corrientes teleinformáticas y en nuevos esquemas de enseñanza" (p. 65). En otras palabras, escenarios que buscan que cada estudiante tenga libertad, en el espacio y en el tiempo, de relacionarse con tutores o maestros del proceso de aprendizaje, por medio de multimedia y aplicativos móviles a través de conexiones, descargas y envíos virtuales, con el fin de generar aprendizaje significativo por el entrecruzamiento de los contenidos educativos, la idoneidad docente y las posibilidades de evaluación. Así, dice Zambrano (2012) que:

El estudiante utiliza una red universitaria que le permite crear, estructurar, compartir y difundir el conocimiento por medio de herramientas como foros, tablón y debate. El formato no presencial da respuesta a los diferentes ritmos de aprendizaje a los estudiantes; se acuerda un plan de trabajo en la que se organizan todas las pruebas; se publican las soluciones y se le informa al discente de su desempeño mediante evaluación y retroalimentación. (p. 68)

Para el análisis e incidencia, Zambrano (2012) clasifica cuatro variables: 1. la materialización y materialidad de los espacios virtuales, traducido en la utilidad que las TIC le aportan al sistema educativo, 2. la construcción y consolidación de una comunidad virtual sin límites grupales que flexibilizan las técnicas didácticas, 3. la disponibilidad permanente en tiempos acordados para abordar necesidades del aprendizaje como las pedagogías, tutorías y clases, y 4. la colaboración asincrónica, el seguimiento y la evaluación continua y la idoneidad de profesores en los procesos educativos.

Esto es lo que ocurre con el mobile learning o aprendizaje móvil, un tipo de enseñanza-aprendizaje que utiliza dispositivos electrónicos móviles, como el celular, y que se caracteriza por la ubicuidad, es decir, que se puede aprender en cualquier 
momento y lugar. El mobile learning o aprendizaje móvil es "la modalidad educativa que facilita la construcción del conocimiento, la resolución de problemas de aprendizaje y el desarrollo de destrezas o habilidades diversas de forma autónoma y ubicua gracias a la mediación de dispositivos móviles portables" (Brazuelo y Gallego, 2012, p. 17).

A partir del concepto de "movilidad" implica: a) "movilidad física", ya que las personas se encuentran en constante movimiento, facilitando su aprendizaje en función de su cotidianidad, b) "movilidad tecnológica", relacionada con el hecho de que se dispone de tecnologías móviles que se pueden llevar al lugar que se quiera y para distintas tareas, con comodidad, economía y facilidad, y c) "movilidad social", lo que significa que se puede aprender "de" y "con" los demás, en diversos entornos, para la superación o mediación de problemáticas socioeconómicas y sociopolíticas (Sharpes, Taylor y Vacuola, 2007, citados en Brazuelo y Gallego, 2012). Movilidad que se aplica en ambos sentidos: docentes y estudiantes.

En este sentido, la apropiación social de las TIC es transferida a la apropiación social de los ámbitos mediados por las mismas, a través de este componente de "movilidad" implícito de los nuevos dispositivos. Tomando de ejemplo Las nuevas tecnologías en niños y adolescentes: guía para educar saludablemente en una sociedad digital, podemos analizar cómo las diferentes tecnologías existentes en la actualidad le ofrecen pautas a padres y educadores para la correcta incorporación de las TIC en la formación y crianza de menores, desde una dimensión que favorece el desarrollo integral y saludable (Roca, 2015).

Para que la apropiación social pueda darse, se requiere establecer un enfoque de largo alcance y larga duración, que implique el seguimiento de los usos y prácticas tecnológicas, además de sus constantes mutaciones y cargas simbólicas. La apropiación social de TIC se entiende con mayor precisión en la perspectiva de Carmen Gómez Mont (2015), que mediada por la visión del historiador, investigador y filósofo francés Michel de Certeu, establece que la tecnología no es el centro del proceso y apropiación del desarrollo sino la materialidad de la persona, la cual evita la alienación, a la vez que está al servicio del ser. En otras palabras, el dispositivo como contra-hegemón y las subjetividades en la cotidianidad.

Esta línea de trabajo pone las TIC al servicio de la formación ciudadana en el sentido de que los sujetos pueden encontrar un nicho de "subalternidad" en las nuevas tecnologías aplicadas y en la educación. Recordemos que este concepto hace referencia, en ciencias sociales, a los sectores marginales, es decir, que, para algunos, la formación ciudadana es un tema marginal que no requiere cuidado.

En el artículo "Los dispositivos móviles en el proceso de aprendizaje", Basantes et al. (2017) propusieron determinar la utilización de los dispositivos móviles en el 
proceso de aprendizaje en la Facultad de Educación Ciencia y Tecnología, de la Universidad Técnica del Norte, ubicada en la ciudad de Ibarra, Ecuador. A través de la investigación bibliográfica, los autores identifican las tenencias de tipo y uso de al menos un dispositivo móvil, para luego analizar e interpretar el impacto de la utilidad de estos dispositivos en la educación, concluyendo que, "el uso de los dispositivos móviles potencia la interacción dentro y fuera del aula estimulando la exploración, la comunicación, el pensamiento crítico y reflexivo" (p.79).

Lo anterior, remonta a las discusiones modernas que posibilitaron el surgimiento de teorías del aprendizaje, como la teoría conversacional o de la actividad, la del aprendizaje situado, el constructivismo social, el conectivismo o el aprendizaje colaborativo. Estas discusiones tienen como punto de partida los postulados de grandes interventores del ámbito educativo, desde diferentes perspectivas como la del psicólogo sociocultural Lev Vygotsky (1978) en sus planteamientos de pensamiento y lenguaje en el desarrollo. Y así como el bio-poder foucaultiano sirve para entender los dispositivos hoy, estas teorías del aprendizaje y la educación pueden generar posibilidades de entender la potencialidad del uso del celular como aliado importante en la docencia y los procesos de aprendizaje.

El teléfono celular es un medio de comunicación con gran poder de influencia en todos los aspectos sociales, gracias a su ubicuidad, personalización y capacidad para crear redes sociales, hace que las personas interactúen de múltiples maneras, con dispositivos y tecnologías que trascienden el espacio y el tiempo, y que ocupan parte importante de la vida cotidiana de usuarios y consumidores. Además, otra característica del celular es la gran cantidad de patologías derivadas de su uso constante, como es el denominado autismo tecnológico (Hernández, 2013).

En el estudio "Tecnologías emergentes al servicio de la educación", se puede notar cómo influye la tecnología en la educación, en especial las tecnologías emergentes como la realidad aumentada y el aprendizaje basado en juegos (Medina, 2012). Las tecnologías del aprendizaje móvil son un ámbito de las TIC, enfocado en educación y procesos de formación. En "[r]ecursos móviles y plataformas virtuales en pro de la educación", Ayala y Vallejo (2012) hacen referencia a la búsqueda de nuevos escenarios formativos y recursos tecnológicos didácticos, que puedan enriquecer los procesos de enseñanza y aprendizaje en las aulas, ya que, según Ayala y Vallejo (2012):

La educación no se refiere solamente a la escuela y el aprendizaje, esta no es un proceso exclusivo de un entorno físico en particular. Los cambios educativos dados a partir de la integración en la enseñanza de las Tecnologías de la Información y Comunicación - TIC, propias del siglo XXI, han incidido en la manera como se accede a la información, las formas de relacionamiento entre los docentes y estudiantes". (p. 115) 
El juego hace parte de nuestra vida, y para el caso de la educación con apoyo en TIC, adquiere importancia, sin desconocer que también deriva en dos términos retadores para la estrategia, estos son: gamificación y edutainment, los cuales, al estar vinculados a la estrategia de microlearning, potencian el ejercicio realizado en la región de Urabá.

\subsection{Hacia una propuesta de formación ciudadana mediada por TIC}

En la identificación de las necesidades educativas, implementación de TIC y formación ciudadana en derechos, la conectividad y el acceso a dispositivos, permite cada vez, y con mayor incidencia, la intervención de contextos. Esto se evidencia en "Ruralidad y dispositivos móviles: apropiación social y uso de la Tableta de Información Cafetera TIC", un estudio de caso de la Federación Nacional de Cafeteros para Antioquia, cuyo objetivo fue identificar el uso y la apropiación de las tabletas TIC como dispositivos en los municipios cafeteros de Antioquia y cómo es posible articularlos al quehacer campesino (Zapata y Marín, 2015).

Ahora bien, también se les puede entender como herramientas que permiten la medición y el seguimiento que se evidencia en el proyecto "Rol de las TIC en la construcción de la ciudadanía digital: Apropiación y uso social de internet", en el cual, la Universidad de Medellín trabajó con jóvenes pertenecientes a las instituciones educativas El Pinal y Félix Henao Botero de la comuna ocho de Medellín, con el fin de analizar los usos que hacen de las TIC; como caso de internet, los estudiantes de la media vocacional de dichas instituciones educativas en los procesos de construcción ciudadana (Torres, 2016).

Ambos estudios revelan la incidencia de las TIC y cómo la integración de los medios tecnológicos permite abordar de manera articulada, las necesidades del contexto, y contribuir de manera efectiva en el fortalecimiento del desarrollo humano y la formación de ciudadanías.

Entrando en materia, podemos insertarnos en un escenario real y plantear un proyecto investigativo que permita la mediación tecnológica para la apropiación social de las TIC y la formación ciudadana de jóvenes en Urabá. Subregión que cuenta con una extensión de $11.799 \mathrm{~km}^{2}$ que representa el 18,54 \% de Antioquia y una población cercana a los setecientos mil habitantes, distribuida en once municipios, con diferentes características económicas, ambientales, culturales y educativas, y un alto nivel de relevancia por las problemáticas que enfrenta. 


\section{Metodología}

La metodología utilizada en esta investigación, es un diseño de tipo no experimental aplicado de forma transeccional o transversal, definido por Hernández et al. (2010) como "la investigación que se realiza sin manipular de manera deliberada las variables. Es decir, se trata de estudios donde no hacemos variar en forma intencional las variables independientes para ver su efecto sobre otras variables" (p. 149). Los autores señalan que los estudios transeccionales, "recolectan datos en un solo momento, en un tiempo único. Su propósito es describir variables y analizar su incidencia e interrelación en un momento dado" (p. 151). El diseño transeccional tuvo un alcance correlacional, que busca "conocer la relación o grado de asociación que exista entre dos o más conceptos, categorías o variables en un contexto en particular" (p. 81). El alcance correlacional de esta investigación se manifiesta al poner en conversación la incidencia de las variables derivadas de la formación ciudadana y de la apropiación social de las TIC, en el contexto de los estudiantes de décimo y undécimo de la IERZ de Necoclí.

La metodología recurrió a técnicas como la encuesta y el análisis integrado de datos. Para la primera, se emplearon dos tipos de cuestionarios, ambos estructurados con preguntas abiertas y cerradas, diseñados con escala Likert. El segundo, por su parte, incluyó preguntas de selección múltiple con una o varias respuestas, ponderación de variable y preguntas abiertas cortas.

En el caso del análisis integrado de datos, se diseñaron las micro sesiones de aprendizaje y se pusieron a prueba con la comunidad de estudiantes, a pesar de la pandemia por la Covid-19, que en un comienzo se presentó como un obstáculo para el proceso, se convirtió en la oportunidad de validar el ejercicio, ya que el diseño de micro sesiones fue una oportunidad para continuar con las actividades académicas en modalidad virtual, potenciando las actividades de micro learning en medio de la situación vivida.

El enfoque mixto fue de tipo anidado o incrustado concurrente de modelo dominante, la concurrencia significa que "Is]e aplican ambos métodos de manera simultánea (los datos cuantitativos y cualitativos se recolectan y analizan más o menos en el mismo tiempo)" (Hernández et al., 2010, p. 546). Por lo tanto, los instrumentos aplicados captaron de la forma más simultánea posible los datos.

Además, al ser anidada o incrustada de modelo dominante, implica que "[e]l método que posee menor prioridad es anidado o insertado dentro del que se considera central. Tal incrustación puede significar que el método secundario responda a diferentes preguntas de investigación respecto al método primario" (Hernández et al., 2010, p. X). De esta manera, el enfoque cualitativo está incrustado dentro del 
enfoque cuantitativo dominante, que es donde reside la mayor cantidad de datos concernientes a la investigación, esto proporciona una visión más amplia de los fenómenos a estudiar.

En cuanto al muestreo es de tipo no probabilístico, hace que "el tamaño de muestra no es importante desde una perspectiva probabilística, pues el interés del investigador no es generalizar los resultados de su estudio a una población más amplia. Lo que se busca en la indagación cualitativa en profundidad" (Hernández et al., 2010, p. 394).

De esta manera, la muestra de la investigación fueron los estudiantes del grado decimo, que cuenten con las condiciones de acceso y conectividad para diligenciar de manera voluntaria los instrumentos de investigación, y los estudiantes del grado once, que participaron de la jornada propuesta para el diligenciamiento en la IERZ del municipio de Necoclí.

Cabe rescatar que, la IER fue seleccionada por criterio de conveniencia, es decir "casos disponibles a los cuales tenemos acceso" (Hernández et al., 2010, p. 401). Por ser una de las instituciones educativas seleccionadas del proyecto "Ecosistema de contenidos digitales para Apps en Urabá" que enmarca la investigación, y se eligió de manera específica esta institución educativa por su carácter rural y por ser parte del municipio de Necoclí, el cual cuenta con una amplia gama de características portuarias, agrícolas, históricas y sociales, lo que hace de Necoclí un territorio que resume toda la región de Urabá en un municipio.

Finalmente, los instrumentos fueron diligenciados por cuarenta estudiantes del grado decimo, que contaron con las condiciones de acceso y conectividad para llenar de manera voluntaria los instrumentos de investigación, y de grado once participaron en la realización de la encuesta la jornada propuesta para en la IERZ del municipio de Necoclí. Previamente se realizó una prueba piloto con cinco estudiantes de los grados decimo y once, lo cuales validaron los instrumentos, emplearon un tiempo promedio de cuarenta y tres minutos, y no señalaron cambios o ajustes de lenguaje, estructura, instructivo o tiempo para su realización.

Como categorías de análisis se plantean:

1. Formación ciudadana: hace referencia a comprender desde un ambiente de convivencia escolar cómo se forjan los comportamientos relacionados con la ciudadanía. Subcategorías: participación ciudadana, convivencia inclusiva, convivencia democrática y convivencia pacífica.

2. Apropiación social de las TIC: rutinas de uso y la relación con los dispositivos móviles en la cotidianidad de los estudiantes. Subcategorías: adquisición de 
dispositivos móviles, rutinas de uso de los dispositivos móviles, temores frente a los dispositivos móviles y aprendizaje móvil.

\subsection{Individualizar al sujeto digital con una propuesta metodológica para la mediación de sus necesidades}

Individualizar al sujeto digital, requiere para la apropiación social poder acceder y hacer uso de las TIC. A medida que avanzamos en la reflexión podemos evidenciar que investigaciones como las citadas, desde lo metodológico o lo teórico, nos permiten entender que las TIC en el aula deben aliarse de forma apropiada con el entorno en el cual se presentan articuladas con las capacidades y configuraciones de la cultura en el territorio.

En el caso de Necoclí, podemos ver grandes brechas, sobre todo en la caracterización de los jóvenes respecto a los casos abordados en Barranquilla y Medellín, al exigir que está propuesta requiera la construcción conjunta con la juventud. Si bien son mayores las fortalezas que aportan estos estudios sobre dispositivos móviles, existen vacíos en cuanto a la implementación de las estrategias en las zonas rurales, por lo que emprender este proyecto genera cabida teórica y práctica en pro de generar más conocimiento frente a la mediación de TIC para la apropiación social en Necoclí, y así aportar de manera significativa e innovadora a la búsqueda de nuevos resultados en el acceso y uso de las TIC a nivel social, educativo y democrático en la formación de sujetos políticos en la juventud.

La investigación permite complementar esos procesos mediados por las TIC, ya que la metodología mixta, entendida desde los planteamientos de Johnson y Onwuegbuzie (2004), es "el tipo de estudio donde el investigador mezcla o combina técnicas de investigación, métodos, enfoques, conceptos o lenguaje cuantitativo o cualitativo en un solo estudio" (p. 17). De suerte que, el repertorio de necesidades cobra sentido desde el enfoque de la "sociedad del conocimiento", al enmarcar la investigación en la lectura social y política, y a partir de ahí generar producción tecnológica que trabaje la formación ciudadana.

Lo contextual en la aplicación de las herramientas, como en los productos y prácticas derivados en el proceso de investigación, parten del seno de la realidad social y vuelvan a ella. Con esta perspectiva, una aproximación al aprendizaje móvil desde autores como Crompton y Traxler (2015), que definen el aprendizaje mediante el análisis de contextos, la interacción social y la interacción con el contenido, utilizando equipos electrónicos portátiles, incluye y trata de manera simbiótica tres elementos relacionados con el aprendizaje como los contextos, las tecnologías y los contenidos; logrando con esto la diversificación del contexto, la mediación con tecnologías móviles y el desarrollo de contenidos digitales educativos. 
Sobre la articulación de tecnologías de información y comunicación TIC a las prácticas de enseñanza y de aprendizaje, debemos atender las dificultades que ya la literatura reseñada muestra, como la reticencia de los docentes frente al cambio en sus prácticas y la "tecnofobia", entendida como el temor de ser reemplazados por las tecnologías, de la instrumentalización de la práctica pedagógica, la lógica institucional de invertir en artefactos más que en gramáticas nuevas, entre otros (EAV, 2006, p. 16).

\section{Resultados}

\subsection{Entre los hallazgos se destaca:}

- La prevalencia de uso de dispositivos móviles en la región por encima de computadores, portátiles y tablets; sin excepción, todos tienen un móvil, propio o de la familia en casa.

- Hay una dedicación entre una y dos horas a labores educativas en el móvil, tiempo de consumo digital con atención y concentración real.

- La mayoría de los usuarios más comprometidos con el proceso educativo en móviles, son chicos entre los doce y quince años.

- La prioridad que dan a realizar sus obligaciones académicas por encima de otras actividades.

\subsection{En relación con la formación ciudadana se encontró:}

- Dificultades e impedimentos para una real participación, que garantice democracia plena.

- Ambiente propicio para la apatía política, en gran medida por el desconocimiento que tienen desde sus derechos y deberes, y porque observan a sus gobernantes como personas distantes.

En la figura 1 se destacan los medios por los cuales los estudiantes reciben información acerca de la política en el país.

De la figura 1 se concluye que, entre los medios que contribuyen a la formación de ideas sobre la política, el primer lugar es para la televisión, seguido por las redes sociales. Lo que da cuenta de las de diferentes circunstancias entre un grupo y el otro; el primer grupo recibe los temas desde lo que establece un canal donde el usuario es solo un televidente, mientras que en el segundo abunda la información. Sin embargo, a la vez que el usuario puede ser generador de contenido o seguidor se permite una comunicación en doble vía con el medio. 


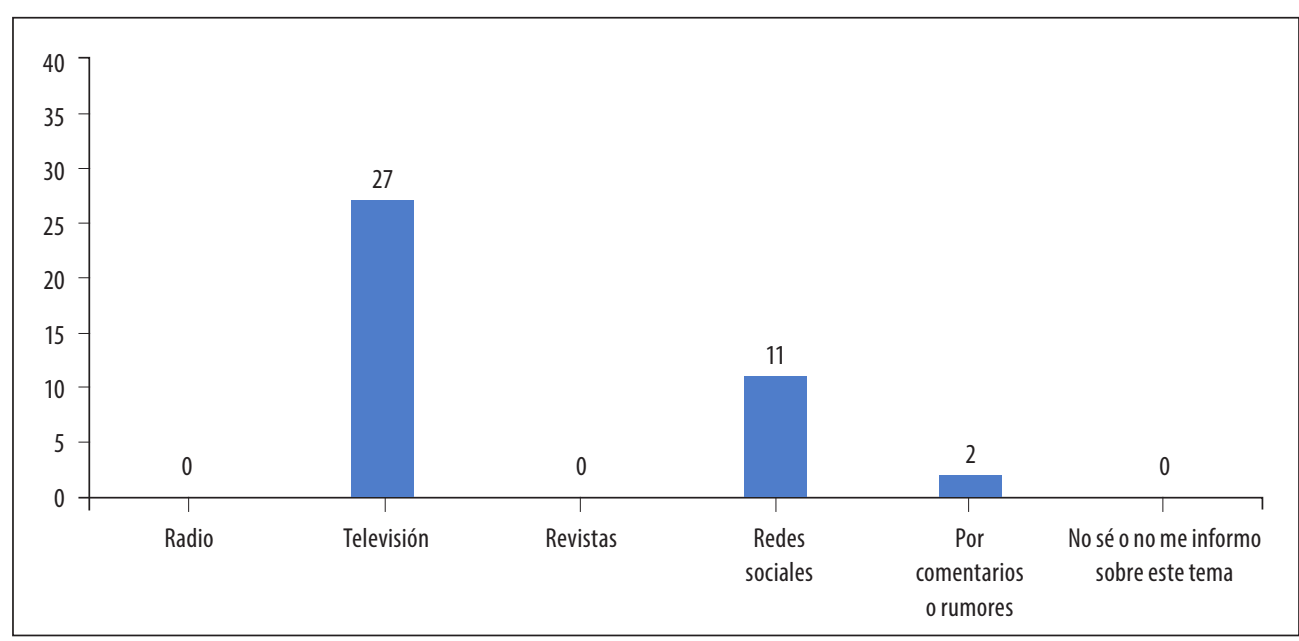

Figura 1. Medios para enterarse sobre política

Fuente: elaboración propia

Además, la suma del contenido mediático que producen los medios de comunicación y el contexto en el cual viven y se apropian de su identidad y tradiciones, son factores que contribuyen a forjar los principios propios para la vivencia en sociedad, como los que se destacan en la figura 2.

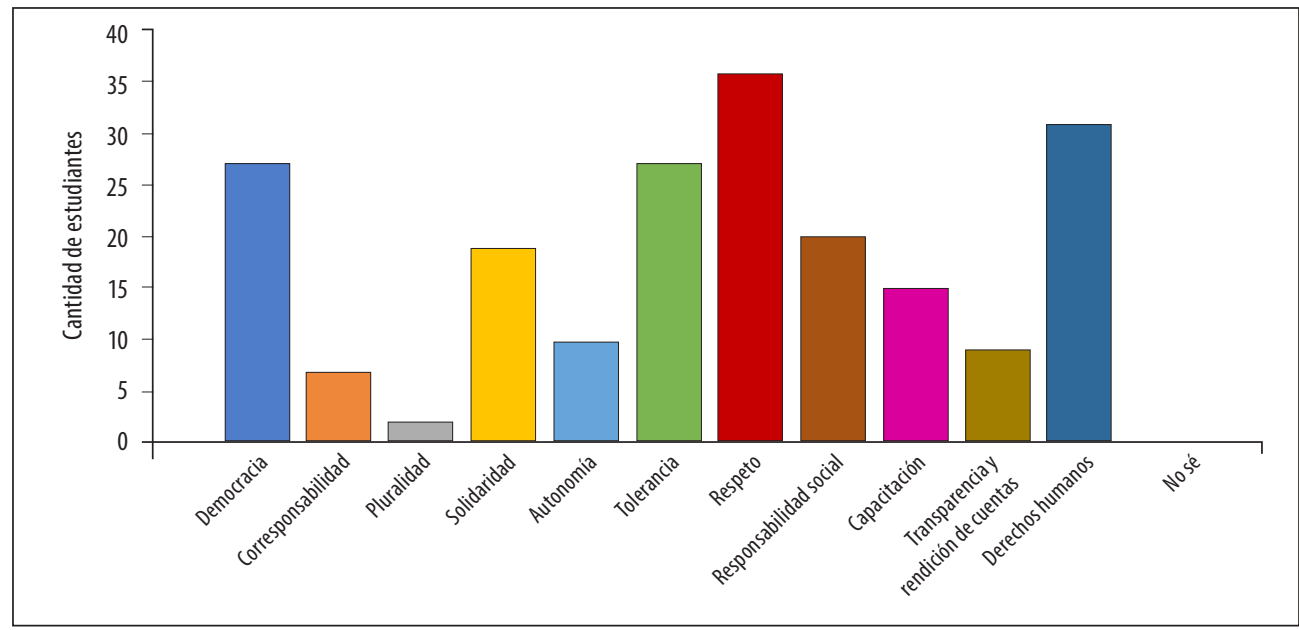

Figura 2. Principios más importantes para la convivencia Fuente: elaboración propia

La figura 2 evidenciamos que el respeto, la tolerancia, los derechos humanos y la democracia, son los valores fundamentales para la convivencia. Mientras la pluralidad, 
la corresponsabilidad y transparencia, son los de menor valor, pero a su vez tienden a ser más cotidianos.

Este ejercicio también permite reconocer a la escuela como como un espacio de diálogo abierto mediado por los docentes en temas amplios, con el fin de prevenir la exposición irracional a riesgos y promover la construcción de posturas ante los diferentes temas que se dan en la sociedad con argumentos sólidos y criterios claros.

Finalmente, se aprovechan los hallazgos del proyecto investigación macro "[e]cosistema de contenidos digitales para apps en Urabá", y se propone una alternativa de micro aprendizaje o microlearning, direccionado a la comunidad académica para la formación de ciudadanía por medio de micro sesiones, expresión más pequeña a la que se puede llevar el proceso de aprendizaje, en un área de estudio concreta (Palazón, 2014), que evidencia la potencialidad de la propuesta asumida por Eduapps.

\section{Discusión y Conclusiones}

- Si bien las instituciones suelen estar dotadas de tecnología como parte de iniciativas de orden digital de los diferentes mandatarios en sus programas del gobierno, la zona no cuenta con señal estable de red, lo que impide una buena conectividad.

- El ejercicio de investigación da cuenta de cómo la apropiación social de tecnologías móviles son una oportunidad para la formación ciudadana, responde a un modelo de ecosistema de contenidos digitales que propende por una cercanía a experiencias de enseñanza y aprendizaje, por medio de dispositivos móviles, reconociendo que hay por lo menos uno en cada casa.

- La apuesta es fortalecer la formación ciudadana entre estudiantes de los últimos años de bachillerato, etapa en la que se afinan las orientaciones políticas de los individuos. Se priorizó este tema por la necesidad de crear tejido políticosocial en la zona.

- La posibilidad de utilizar las tecnologías móviles y los contenidos elaborados para Eduapps, permite transversalizar información que fortalece el aprendizaje de los estudiantes mediante micro cursos, según el modelo Eduapps en este caso de formación ciudadana.

- El aprovechamiento del ecosistema y de las herramientas dispuestas contribuyen a la cercanía de docentes y alumnos en función de conocimientos transversales al currículo que por su facilidad de acceso propician un panorama más amplio de formación.

- El microlearning es una oportunidad para el encuentro y la construcción territorial, a partir de lo local en los procesos de aprendizaje digital se refuerza lo identitario. 
- La posibilidad de estar conectados da la sensación de ser ciudadanos del mundo, ya que la red permite inmersiones en búsquedas y relaciones construidas con sus pares y docentes en el ecosistema que propiciará la creación de redes de interés a futuro.

- Es una oportunidad para que los jóvenes tengan una ventana al mundo, a la vez que permite que la identidad e idiosincrasia del territorio se muestre y tenga una relación de emisor y receptor con la actualidad.

- En consecuencia, es pertinente el estudio de las categorías de la educación ciudadana basado en los comportamientos de los jóvenes en sociedad, en el que la escuela es un escenario de convivencia y muestra de los valores ciudadanos de cada uno de los integrantes de esta, con el fin de crear salidas medias por las TIC y versados por procesos de apropiación que sean propios de cada comunidad.

- En cuanto a los retos que tiene la formación ciudadana en un escenario de apropiación, es importante resaltar que la diversidad y la inclusión son el epicentro del estudio, ya que el flagelo de la guerra común a todos los habitantes, sumado a los diferentes grupos culturales hace que las perspectivas sobre la ciudadanía desde aspectos como la convivencia, la paz y la democracia tengan matices diferentes. Sin perder de vista lo que tienen en común como el uso de las redes sociales, la televisión nacional y en menor medida la radio.

- Por otro lado, el municipio sobre el cual se realizó la investigación será intervenido por ambiciosos proyectos portuarios, cabría entonces ver si el intercambio cultural producido por las TIC se ve influenciado por el intercambio cultural producido por las dinámicas portuarias, y cómo esto se ve reflejado en la vivencia de la ciudadanía.

- A partir de esta temática, pueden surgir otras de interés para micro cursos elaborados en contexto de región como los referentes al tema de paz o de memoria, de tal manera que contribuyan a la construcción de una zona más pacífica, incluyente y participativa.

- El ejercicio de investigación en pandemia obligó al equipo a idear de forma creativa la estrategia para continuar con la formación de maestro, ya sea para implementar el ecosistema y probarlo, o para crear en nuevos micro cursos una oportunidad en zonas alejadas.

- La investigación "Macro Ecosistema de contenidos digitales para Apps Eduapps", no pretendía resolver los problemas tecnológicos, ni educativos, y mucho menos sociales de la región de Urabá, su apuesta era por el diseño de una metodología de cocreación que permitiera idear contenidos digitales para aplicaciones que 
fueran materiales elaborados por los docentes desde su lugar, con el fin de lograr apropiación fuerte en la formación de los alumnos. Pero los investigadores no contaban con la llegada la Covid-19 en la mitad del proceso y si bien no era el objetivo inicial esto fue la oportunidad para generar no solo contenidos, sino una metodología de cursos cortos denominados microlearning que cambiarían la mentalidad de los docentes al enfrentarse a nuevas formas de trabajo que sin depender de la red internet pero si con la ayuda de los móviles pueden provocar verdaderos cambios en la región y darles mayor autonomía en una formación más acorde con el contexto. La reflexión de este artículo sobre el ejercicio con la Formación ciudadana es solo un ejemplo de lo que se puede crear a futuro.

\section{Referencias}

Ayala, M. y Vallejo, C. (2012). Recursos móviles y plataformas virtuales en pro de la educación. Aprender y educar con las tecnologías del siglo XXI. https://bit.ly/32Xddky

Basantes, A., Naranjo, M., Gallegos, M. y Benítez, N. (2017). Los Dispositivos Móviles en el Proceso de Aprendizaje de la Facultad de Educación Ciencia y Tecnología de la Universidad Técnica del Norte de Ecuador. Formación universitaria, 10(2), 79-88. https://www.scielo.cl/scielo. php?script $=$ sci_arttextEpid $=$ S0718-50062017000200009

Benjamín, W. (1936). La obra de arte en la época de su reproductibilidad técnica. Godot ediciones.

Benjamín, W. (2009). El Narrador. Abada.

Boris, M. (2015). Las competencias digitales en la escuela. Las nuevas tecnologías en niños y adolescentes. Guía para educar saludablemente en una sociedad digital. Hospital Sant Joan de Déu. http://www. codajic.org/sites/www.codajic.org/files/Las\%20nuevas\%20tecnolog\%C3\%ADas\%20en\%20\%20 ni\%C3\%Blos\%20y\%20adolescentes.pdf

Brazuelo, F. y Gallego, D. (2012). Mobile learning. Dispositivos móviles como recurso educativo. Eduforma.

Cardona, O., Chaverra, C. y Restrepo, O. (2016). Caracterización de los estilos de enseñanza que presentan los docentes en relación con los usos de las TIC en el aula. Itesis doctoral, Universidad Pontificia Bolivariana]. Repositorio Institucional UPB. http://bit.ly/2GCKabn

Claros, O. (2013). Matrix del dispositivo móvil. Razón y palabra, 85(18), 281-296. http://www. razonypalabra.org.mx/N/N85/N85/06_Claros_V85.pdf

Crompton, H. y Traxler, J. (2015). Mobile Learning and Mathematics. Foundations, Design, and Case Studies. Routledge.

Díaz Barriga, F. y Hernández, G. (2001). Estrategias docentes para un aprendizaje significativo. Una interpretación constructivista. McGrawHill.

Dussel, I. y Quevedo, L. (2010). Aprender y enseñar en la cultura digital. VI Foro Latinoamericano de Educación. Educación y Nuevas Tecnologías: Los desafíos pedagógicos ante el mundo digital. Editorial Santillana. 
Foucault, M. (1980). Power/Knowledge: Selected Interviews and Other Writings, 1972-1977. Harvester.

Fuentes, R. (2010). Investigación de la comunicación: referentes y condiciones internacionales de un diálogo transversal de saberes. Signo y Pensamiento, 29(57), 38-49. https://www.redalyc. org/articulo.oa?id=86020052003

Gaviria Díaz, C. (2015, 11 de marzo) ¿Cómo educar para la democracia? Conferencia de Carlos Gaviria Díaz a los maestros del Gimnasio Moderno [conferencia]. Gimnasio Moderno, Bogotá, Colombia. https://gimnasiomoderno.edu.co/portfolio-item/como-educar-para-la-democracia-2/

Gewerc, F., Fragga, F. y Roldés, V. (2017). Niños y adolescentes frente a la competencia digital. Revista interuniversitaria de formación del profesorado, 89(31), 171-186.

Gómez Mont, C. (2009). La sociología de los usos: una perspectiva en construcción desde la escuela de pensamiento francófona y anglófona. Sociedad del conocimiento. Propuestas para una agenda conceptual. UNAM

Gómez Mont, C. (2015) La apropiación social del conocimiento. Revistas Universitas Científica, 18(1), $58-61$. https://bit.ly/3560790

Grupo de Investigación en Ambientes Virtuales (EAV). (2006). Tecnología-Comunicación-Educación: la tríada. Un modelo para la educación en ambientes virtuales. Editorial UPB.

Hernández, L. (2013). Onto semiótica. Universidad del Zulia.

Hernández, R., Fernández, C. y Baptista, P. (2010). Metodología de la Investigación. Mc Graw Hill.

Johnson, B. y Onwuegbuzie, A. (2004). Mixed Methods Research: A Research Paradigm Whose Time Has Come. Educational Researcher, 33(7), 14-26. https://bit.ly/2Ic7Tm9

Magendzo, A. (2005). Alteridad y diversidad: componentes para la educación social. Pensamiento educativo. Revista de investigación educacional latinoamericana, 37(2), 106-116.

Maldonado, M. (2004). Formación del ciudadano participativo. Educere, 8(27), 469-474. https:// www.redalyc.org/pdf/356/35602704.pdf

Medina, L. (2012). Tecnologías emergentes al servicio de la educación. Aprender y educar con las tecnologías del Siglo XXI. Colombia digital.

Ministerio de Educación Nacional. (2013). Competencias TIC para el desarrollo profesional docente. http://bit.ly/2GyKyHx

Nkeze, E., Pearce, J. y Womer, M. (2007). Device Description Landscape 1.0.W3C Working Group Note 31 October 2007. https://bit.ly/3pInXwI

Nordin, N., Amin Embi, M. y Yunus, M. (2010) Mobile Learning Framework for Lifelong Learning. Procedia Social and Behavioral Sciences, 7, 130-138. https://doi.org/10.1016/j.sbspro.2010.10.019

Olivé, L. (2007). La ciencia y la tecnología en la sociedad del conocimiento. ética, política y epistemología. Fondo de Cultura Económica. 
Palazón, J. (2014). Aprendizaje móvil basado en micro contenidos como apoyo a la interpretación instrumental en el aula de música en secundaria. Revista de Medios y Educación, (46), 119-136. https://www.researchgate.net/publication/265685372_Aprendizaje_movil_basado_en_ microcontenidos_como_apoyo_a_la_interpretacion_instrumental_en_el_aula_de_musica_ en_Secundaria

Quiroz, R. y Jaramillo, O. (2009). Formación ciudadana y educación cívica: ¿cuestión de actualidad o de resignificación? Enseñanza en ciencias sociales, 8, 97-103. https://www.redalyc.org/articulo. oa?id=324127628011

Roca, G. (2015). Las nuevas tecnologías en niños y adolescentes. Guía para educar saludablemente en una sociedad digital. Hospital San Joan de Déu. https://bit.ly/2UFnvRO

Said, E. (2012). Ciudadanía móvil Apropiación y participación de jóvenes en sectores vulnerables en Colombia. Universidad del Norte Editorial. http://manglar.uninorte.edu.co/ bitstream/handle/10584/5552/9789587411775\%20 eCiudadania\%20 movil. pdf? sequence $=1$ EisAllowed $=y$

Torres, Y. (2016). Rol de las TIC en la construcción de la ciudadanía digital: apropiación y uso social de internet, en jóvenes pertenecientes a la i. e. el pinal y Félix Henao botero de la comuna $n .^{\circ}$ 8. Itesis de maestría, Universidad de Medellín]. Repositorio Institucional Universidad de Medellín. https://repository. udem.edu.co/bitstream/handle/11407/4652/T_MC_11.pdf?sequence = 1

Unesco. (2005). Hacia las sociedades del conocimiento. Unesco.

Van Dijk, J. (2005). Depending Divide, Inequality in the Information Society. Sage publications Inc.

Vygotsky, L. (1978). Pensamiento y lenguaje. Paidós.

Viñals, A. (2016). El ocio conectado, móvil, transmediay multisoporte de los jóvenes en la era digital. Fonseca, Journal of Communication, (13), 99-1 13. http://revistas.usal.es/index.php/2172-9077/article/view/ fjc20161399113/15783

Zambrano, W. (2012) Modelo de aprendizaje virtual. ECOE Ediciones

Zapata, M. y Marín, B. (2015). Ruralidad y dispositivos móviles: apropiación social y uso de la Tableta de Información Cafetera TIC. Estudio de caso Federación Nacional de Cafeteros para Antioquia. Revista Lasallista de Investigación, 12(2), 19-27. https://www.redalyc.org/ pdf/695/69542291003.pdf 